\title{
ON A CLASS OF GEOMETRY-DRIVEN FREE BOUNDARY PROBLEMS*
}

\author{
DARREN CROWDY ${ }^{\dagger}$
}

\begin{abstract}
An abstract class of two-dimensional geometry-driven free boundary problems is considered in which the evolution of the Cauchy transform of the time-evolving domain satisfies a partial differential equation of conservation type. This idea generalizes an association between free boundary problems and the inverse gravitational problem. It is shown that this class of free boundary problems admits exact solutions expressible in terms of a finite set of time-evolving parameters. In addition, even when exact solutions are not available, a theorem is established which shows that solutions corresponding to certain initial conditions possess nontrivial conserved quantities. Two distinct problems of physical interest are shown to fall within this abstract class: the evolution of a fluid blob in a rotating Hele-Shaw cell and the evolution of highly viscous fluid under the effects of surface tension. Various aspects of these two problems are discussed.
\end{abstract}

Key words. free boundary problems, sintering, Hele-Shaw, Cauchy transforms

AMS subject classifications. $30,35,76$

PII. S0036139999357988

1. Introduction. Consider a time-evolving region of fluid $D(t)$. Now define the Cauchy transform $C(z, t)$ of the fluid domain $D(t)$ as

$$
C(z, t)=\frac{1}{\pi} \iint_{D(t)} \frac{d x^{\prime} d y^{\prime}}{z^{\prime}-z}
$$

A physical interpretation of the Cauchy transform is in terms of gravitational potentials; note that

$$
C(z, t)=-\frac{\partial}{\partial z} \frac{2}{\pi} \iint_{D(t)} \log \left|z-z^{\prime}\right| d x^{\prime} d y^{\prime}
$$

where the double integral in (2) is the two-dimensional gravitational potential of a uniform mass occupying the region $D(t)$, and so the Cauchy transform can be interpreted as the gravitational force due to that mass at the point $z$.

It has been known for some time that certain classes of free boundary problems involving singularity-driven Hele-Shaw flows can be tackled by first deducing explicit expressions for the Cauchy transform $C(z, t)$ of the fluid domain [22], [23]. In this problem a known distribution of flow singularities (e.g., sources or sinks) explicitly determines the Cauchy transform of the evolving fluid domain. The free boundary problem then reduces to the mathematically analogous inverse gravitational potential problem: Can the shape $D(t)$ of a domain be reconstructed from known gravitational information $C(z, t)$ in the far field?

The inverse gravitational problem has been well-studied and continues to provide an important theoretical perspective for understanding the analogous free boundary problem [13], [18]. Recently, for example, Tian has used a Cauchy integral approach

\footnotetext{
* Received by the editors June 21, 1999; accepted for publication (in revised form) February 5, 2001; published electronically January 18, 2002. This work was supported by National Science Foundation grants DMS-9803167 and DMS-9803358.

http://www.siam.org/journals/siap/62-3/35798.html

$\dagger$ Department of Mathematics, Imperial College of Science, Technology and Medicine, 180 Queen's Gate, London, SW7 2BZ, UK (d.crowdy@ic.ac.uk).
} 
to the sink-driven Hele-Shaw problem to derive results on the breakdown of solutions when surface tension is nonzero [29] and to establish short-time existence and uniqueness of classical solutions to the zero surface tension problem when the initial boundary shapes are analytic [28].

In this paper, we extend the association of free boundary problems with the inverse gravitational potential problem by considering a generalized inverse gravitational problem. This generalized problem corresponds to an abstract class of geometrydriven free boundary problems for which the Cauchy transform of the domain is not determined explicitly by a distribution of imposed flow singularities but instead satisfies a partial differential equation of conservation type. The free boundaries in these problems are not driven by an imposed distribution of singularities but rather by their own intrinsic geometry.

The reason for considering this class of problems from an abstract point of view is predicated on the fact that two well-studied free boundary problems of physical importance, which have been the subject of much recent investigation, fall within this abstract class. Interestingly, this class can include not only Laplace-governed free boundary problems but also biharmonic-governed flows. In section 4 it is shown that both the harmonic-governed problem of the evolution of a blob of fluid in a rotating Hele-Shaw cell evolving under the effects of centrifugal forces and the biharmonicgoverned problem of the sintering of a viscous droplet evolving under the effects of surface tension fall within the abstract class. Given that the two problem statements are so different both mathematically and physically, this mathematical connection is quite unexpected but can provide useful insights and is one of the important new results of this paper.

The fact that both of the above physical problems fall within this abstract class leads to the sharing of certain mathematical properties. For example, both admit classes of exact solutions for the evolution of a simply connected blob of fluid. The exact solutions themselves are not new; such solutions were first found for the rotating Hele-Shaw problem by Entov, Etingof, and Kleinbock [10] and for the viscous sintering problem by Hopper [14], [15] (who used methods very different from those employed here). However, while the exact solutions for simply connected fluid domains might already be known, the unexpected mathematical connections between the two problems (as demonstrated herein) have led the present author to establish new results [8] for the evolution of a fluid annulus in a rotating Hele-Shaw cell-a problem of much recent experimental interest [2], [3], [4]. The new solutions for rotating annuli in Hele-Shaw cells are intimately related (as seen by the results herein) to a class of exact solutions for sintering viscous annuli found by Crowdy and Tanveer [6]. It is clear that an understanding that two problems share an underlying analytical structure can lead to a fruitful cross-collateralization where results from the study of one problem can be imported into another.

For the purposes of the present paper, we restrict consideration to simply connected fluid regions. However, it is important to point out that this study is the first to formulate the viscous sintering problem using the mathematical perspective of Cauchy transforms (although this perspective is naturally related to a formulation in terms of quadrature identities recently discussed by the present author [7]). This formulation is potentially important from the point of view of generalizing exact solutions to the viscous sintering problem (and, indeed, the rotating Hele-Shaw problem) to the case of domains with connectivity greater than two.

A further consequence of the shared mathematical structure is that both problems 
admit certain nontrivial conserved quantities as a result of what is herein referred to as a "circulation theorem." This theorem yields many previously unrecognized conserved quantities for both problems, including cases where exact solutions are not available. These conserved quantities might be useful for checking the accuracy of numerical codes.

Given all the mathematical similarities, it is also important to emphasize that the two problems do have some important differences. Such distinctions are made in section 5 by means of a series of examples. It turns out that the rotating Hele-Shaw problem has additional mathematical structure not shared with the viscous sintering problem - a fact which leads to wider classes of exact solutions. The paradigm of an initial ellipse as it evolves under the dynamics of both problems is an instructive example presented in detail in section 5 . First we demonstrate the (previously unknown) result that the rotating Hele-Shaw problem admits an exact solution for this initial condition. The sintering problem does not. Even so, it turns out to be possible to use the new approach using Cauchy transforms to propose an approximation scheme for the boundary evolution of a sintering ellipse. A low-order, finite-dimensional approximation of the exact dynamics is found to agree very well with full numerical simulations using boundary integral methods [19].

2. A Riemann-Hilbert problem. Consider the following Riemann-Hilbert problem. On the closed analytic curve $\partial D(t)$ bounding a simply connected fluid region $D(t)$,

$$
\bar{z}=C_{i}(z, t)-C_{o}(z, t),
$$

where $C_{i}(z, t)$ is analytic inside $D(t)$ and $C_{o}(z, t)$ is analytic outside $D(t)$. This is a standard Riemann-Hilbert problem, and it is well known [1] that the solution for $C_{o}(z, t)$ can be written as the following integral:

$$
C_{o}(z, t)=\frac{1}{2 \pi i} \oint_{\partial D(t)} \frac{\bar{z}^{\prime} d z^{\prime}}{z^{\prime}-z}
$$

where $z=x+i y$ is taken outside $D(t)$. By Green's theorem [1], this becomes

$$
C_{o}(z, t)=\frac{1}{\pi} \iint_{D(t)} \frac{d x^{\prime} d y^{\prime}}{z^{\prime}-z}
$$

The function $C_{o}(z, t)$ is exactly the Cauchy transform of the domain $D(t)$ as defined in (1). Henceforth, the subscript will be dropped and it will be called $C(z, t)$.

Suppose we now introduce the conformal map $z(\zeta, t)$ from the unit circle in a parametric $\zeta$-plane to the region $D(t)$. Such a function exists by the Riemann mapping theorem. Then (3) can be written

$$
\bar{z}\left(\zeta^{-1}, t\right)=C_{i}(z(\zeta, t), t)-C_{o}(z(\zeta, t), t) .
$$

As an equation relating functions of $\zeta,(6)$ holds everywhere that the functions can be analytically continued. The composed function $C_{i}(z(\zeta, t), t)$ is analytic everywhere in the unit circle, so (6) indicates that the singularities of $\bar{z}\left(\zeta^{-1}, t\right)$ are determined by those of the Cauchy transform $C_{o}(z(\zeta, t), t)$ inside $D(t)$. If the Cauchy transform is known to be meromorphic inside $D(t)$ (with poles of known position and strengths), then the corresponding conformal map will be a rational function, and a comparison of principal parts at the poles yields a set of equations for the conformal mapping 
parameters. This method has been used by a number of authors in the study of variants of the Hele-Shaw problem [22], [23], [24], [10], [11], [12].

This paper generalizes this approach in a different direction and demonstrates that similar ideas can be applied to wider classes of free boundary problems - not just those governed by a Laplace-type field equation, or just singularity-driven flows. It will be shown how the methodology can be applied to study curvature-driven, biharmonic-governed problems such as the viscous sintering of fluid blobs.

3. A class of free boundary problems. We introduce a generalized inverse gravitational problem. Suppose that the Cauchy transform of a uniform gravitating mass $D(t)$ is not known explicitly but instead is known, everywhere outside $D(t)$, to satisfy a partial differential equation of conservation type having the general form,

$$
\frac{\partial C}{\partial t}+\frac{\partial I}{\partial z}=0, \quad C(z, 0)=\text { given }
$$

where $I(z, t)$ is the function, analytic outside $D(t)$, defined by the inhomogeneous Cauchy transform

$$
I(z, t)=\frac{1}{\pi} \iint_{D(t)} \frac{\sigma\left(z^{\prime}, t\right) d x^{\prime} d y^{\prime}}{z^{\prime}-z}
$$

for some known function $\sigma(z, t)$ assumed analytic in $D(t)$. The function $I(z, t)$ corresponds to the solution $I_{o}(z, t)$ of the following Riemann-Hilbert problem:

$$
I_{i}(z, t)-I_{o}(z, t)=\sigma(z, t) \bar{z} \quad \text { on } \partial D(t),
$$

where again $I_{i}(z, t)$ and $I_{o}(z, t)$ denote functions analytic inside and outside $D(t)$, respectively. Following the gravitational interpretation of $C(z, t)$, the function $I(z, t)$ might be interpreted as the force due to a nonuniform mass density $\sigma(z, t)$ occupying the region $D(t)$.

The above specification can refer equally well to a free boundary problem: Define an abstract class of free boundary problems to be those in which the Cauchy transform $C(z, t)$ of the time-evolving domain $D(t)$ satisfies (7) for some choice of $\sigma(z, t)$. Different free boundary problems within this abstract class correspond to different choices of the analytic function $\sigma(z, t)$.

Now consider points $z$ inside $D(t)$ and assume that the boundary $\partial D(t)$ is an analytic curve. The integrals (1) and (8) defining $C(z, t)$ and $I(z, t)$ outside $D(t)$ are nowhere analytic inside $D(t)$. However, suppose that $C(z, t)$ and $I(z, t)$ can be extended as analytic functions into the domain $D(t)$. In general, these continuations will have singularities inside $D(t)$. Tian [28] has shown that $C(z, t)$ can be analytically continued inside $D(t)$ if and only if $\partial D(t)$ is an analytic curve (which has been assumed). The same is true of $I(z, t)$. By the continuation principle [1], (7) is the equation satisfied by these analytic continuations of $C(z, t)$ and $I(z, t)$ for points $z \in D(t)$.

If $\partial D(t)$ is an analytic curve, then there exists a Schwarz function $S(z, t)[9]$ of $\partial D(t)$ defined by

$$
S(z, t)=\bar{z} \quad \text { on } \partial D(t) .
$$

$S(z, t)$ is analytic in an annular region surrounding $\partial D(t)$. In terms of this Schwarz function, the Plemelj formulae [1] can be used to deduce that

$$
\begin{aligned}
C(z, t) & =C_{i}(z, t)-S(z, t), \\
I(z, t) & =I_{i}(z, t)-\sigma(z, t) S(z, t) .
\end{aligned}
$$


Equation (12) reveals that $C(z, t)$ and $I(z, t)$ possess exactly the same distribution of singularities inside $D(t)$ although the singularities of $I(z, t)$ have different strengths owing to the multiplication by the analytic function $\sigma(z, t)$.

The following theorem reveals something about the singularity structure of the Schwarz function $S(z, t)$ inside $D(t)$ under evolution.

THEOREM 3.1. If $C(z, 0)$ is initially such that it has a finite set of isolated (polar or branch-point) singularities at points $z_{j}(0)(j=1, \ldots, N)$ inside $D(0)$ and if $C(z, t)$ satisfies (7) outside $D(t)$ for some $\sigma(z, t)$ analytic in $D(t)$, then (provided a solution exists) $C(z, t)$ continues to possess a finite set of isolated (polar or branch-point) singularities at points $z_{j}(t)(j=1, \ldots, N)$ inside $D(t)$. Furthermore, the singularities move according to the ordinary differential equation

$$
-\dot{z}_{j}(t)+\sigma\left(z_{j}(t), t\right)=0 .
$$

Proof. First note that (8) can be written in the form

$$
\begin{aligned}
I(z, t)=\frac{1}{\pi} \iint_{D(t)} \frac{\sigma\left(z^{\prime}, t\right) d x^{\prime} d y^{\prime}}{z^{\prime}-z} & -\frac{1}{\pi} \iint_{D(t)} \frac{\sigma(z, t) d x^{\prime} d y^{\prime}}{z^{\prime}-z} \\
& +\frac{1}{\pi} \iint_{D(t)} \frac{\sigma(z, t) d x^{\prime} d y^{\prime}}{z^{\prime}-z}
\end{aligned}
$$

or, equivalently,

$$
I(z, t)=\frac{1}{\pi} \iint_{D(t)} \frac{\sigma\left(z^{\prime}, t\right)-\sigma(z, t)}{z^{\prime}-z} d x^{\prime} d y^{\prime}+\sigma(z, t) C(z, t) .
$$

Define the function $\Sigma(z, t)$ as

$$
\Sigma(z, t)=\frac{1}{\pi} \iint_{D(t)} \frac{\sigma\left(z^{\prime}, t\right)-\sigma(z, t)}{z^{\prime}-z} d x^{\prime} d y^{\prime} .
$$

The singularity of the integrand in (16) is removable so that $\Sigma(z, t)$ is deduced to be analytic in $D(t)$. Thus,

$$
I(z, t)=\Sigma(z, t)+\sigma(z, t) C(z, t)
$$

which, when substituted into (7), yields the following partial differential equation for the Cauchy transform:

$$
\frac{\partial C}{\partial t}+\sigma(z, t) \frac{\partial C}{\partial z}+\frac{\partial \sigma}{\partial z}(z, t) C+\frac{\partial \Sigma}{\partial z}(z, t)=0, \quad C(z, 0)=\text { given. }
$$

By the continuation principle, (18) is the relevant equation for the analytic continuation of $C(z, t)$ inside $D(t)$. However, inside $D(t)$, (18) has the form of a first-order linear equation for $C(z, t)$ with coefficients that are known a priori to be analytic in $D(t)$. Solutions for $C(z, t)$, if they exist, will therefore have the same analytic structure inside $D(t)$ as solutions of a first-order linear partial differential equation with analytic coefficients. In particular, using the well-known theory of such equations, it can immediately be deduced that polar and branching singularities are preserved and move on characteristics, i.e.,

$$
-\dot{z}_{j}(t)+\sigma\left(z_{j}(t), t\right)=0 .
$$


The theorem is proved.

Corollary 3.2 (exact solutions). If the Cauchy transform $C(z, 0)$ of a simply connected region $D(0)$ is initially meromorphic inside $D(0)$, then $C(z, t)$ remains meromorphic in $D(t)$ under evolution and the abstract class of free boundary problems (7) admits exact solutions in which the conformal map $z(\zeta, t)$ from a unit $\zeta$-circle to the fluid domain $D(t)$ satisfying $z(0, t)=0$ is a rational function of $\zeta$.

Proof. From Theorem 3.1 we deduce that a Cauchy transform that is initially meromorphic inside $D(0)$ remains meromorphic in $D(t)$ under evolution. Using (12), it can be deduced that the Schwarz function $S(z, t)$ of $\partial D(t)$ is meromorphic inside $D(t)$. Using a standard theorem from the theory of Schwarz functions [9], the result on the rational function form of the conformal mapping function follows immediately (see Chapter 14 of [9] for more details). As discussed in section 2, the evolution of the poles and zeros of the mapping function can be found, for example, by equating principal parts in (6).

3.1. A circulation theorem. We now show that the conservation-form of the evolution equation for $C(z, t)$ combined with the special structure of the flux function $I(z, t)$ leads to the existence of some conserved quantities for the dynamics.

TheOREM 3.3. Consider a closed contour $\gamma$ fixed strictly inside a fluid region $D(0)$. Pick a point $z_{0}$ on $\gamma$ and suppose that the Cauchy transform $C(z, 0)$ of the domain has a single-valued analytic continuation around the path defined by $\gamma$, beginning and ending at $z_{0}$. Then, at least for sufficiently short times so that $\gamma$ remains strictly inside $D(t)$, the circulation-type quantity

$$
\oint_{\gamma} C(z, t) d z
$$

is conserved by the dynamics.

Proof. Consider

$$
\frac{d}{d t} \oint_{\gamma} C(z, t) d z=\oint_{\gamma} \frac{\partial C(z, t)}{\partial t} d z
$$

where we have used the fact that the contour $\gamma$ is fixed. However, (7) implies

$$
\oint_{\gamma} \frac{\partial C(z, t)}{\partial t} d z=-\oint_{\gamma} \frac{\partial I(z, t)}{\partial z} d z=-[I(z, t)]_{\gamma} .
$$

Provided sufficiently short times have passed so that all the singularities that were initially inside $\gamma$ are still inside $\gamma$ (note from (19) that singularities move at finite speed) and that $\gamma$ is still strictly inside $D(t)$, we can deduce

$$
\frac{d}{d t} \oint_{\gamma} C(z, t) d z=-[I(z, t)]_{\gamma}=0
$$

because $I(z, t)$ is a single-valued function of $z$ around $\gamma$, a fact which follows from (12), valid for the analytic continuation of $I(z, t)$ inside $D(t)$, and use of the fact that $C(z, t)$ has, by assumption, a single-valued analytic continuation around $\gamma$. Recall that (12) shows that $I(z, t)$ has the same singularity distribution inside $D(t)$ as $C(z, t)$ (although with different strengths), and so $I(z, t)$ also has a single-valued analytic continuation around $\gamma$. Thus

$$
\oint_{\gamma} C(z, t) d z
$$




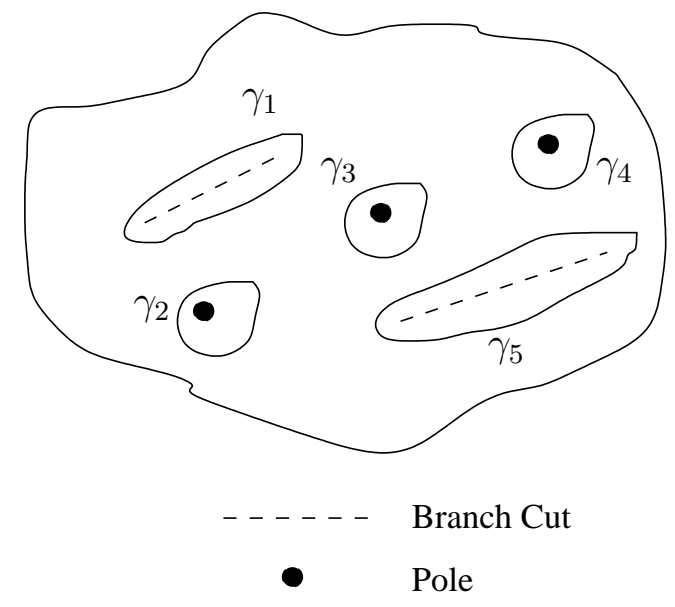

FIG. 1. Singularity structure of $C(z, 0)$ for an example initial blob.

is a quantity conserved by the dynamics.

Remark. If $\gamma$ is chosen so that it encloses a set of singularities of $C(z, t)$, then the circulation-type quantity (24) will, in general, be a nontrivial conserved quantity.

Remark. If the curve $\gamma$ is chosen to be uniformly close to the boundary of the fluid region so that it encloses all singularities of $C(z, t)$, the associated conserved quantity is precisely the area of the fluid domain. Other choices of $\gamma$ enclosing, for example, a subset of isolated singularities, will in general produce independent (and nontrivial) quantities conserved by the dynamics.

Remark. In the special case of the exact solutions in which $C(z, t)$ is meromorphic inside $D(t)$ with a distribution of $N$ poles at points $z_{j}(t), j=1, \ldots, N$, the circulation theorem states that there exist $N$ independent conserved quantities which result from choosing $\gamma$ to encircle each isolated singularity $z_{j}, j=1, \ldots, N$, individually. If the contour surrounding only the singularity at $z_{j}$ is denoted $\gamma_{j}$, the associated conserved quantity is

$$
\oint_{\gamma_{j}} C(z, t) d z
$$

Remark. As an example, consider an initial domain $D(0)$ which has a Cauchy transform $C(z, 0)$ having a distribution of isolated polar and branch-point singularities as shown in Figure 1. In order that $\partial D(0)$ be a closed, analytic curve, a choice of branch cuts within $D(0)$ must be made. Suppose they are as given in Figure 1. Then Theorem 3.3 states that there are five nontrivial conserved quantities associated with this free boundary problem given by

$$
\oint_{\gamma_{j}} C(z, t) d z
$$

where $\gamma_{1}, \ldots, \gamma_{5}$ are shown in Figure 1. The contours $\gamma_{j}$ are chosen to either encircle the poles or each choice of branch cut. Note that the existence of these conserved quantities has been established without knowing any details of the solution, and certainly without knowledge of an exact form of the solution, which may not even exist. Indeed, for general $\sigma(z, t)$, the free boundary problem evolving from an initial condition such as that in Figure 1 (where there are branching singularities and not just 
poles) will not, in general, admit an exact solution describable in terms of a finite set of parameters.

4. Two physical examples. The previous sections considered an abstract class of free boundary problems. We now show that two well-studied free boundary problems of physical interest fall within this class of flows. The following two problems are "geometry-driven" in that their evolution at any time is determined purely by its configuration at that time (and not, for example, by some distribution of driving singularities).

4.1. A droplet in a rotating Hele-Shaw cell. Consider a simply-connected blob $D(t)$ of fluid in a Hele-Shaw cell which will be assumed to be rotating with a (possibly time-dependent) angular velocity $\omega(t)$. This problem has been well-studied over the last few years, numerically [27], analytically [10], and experimentally [2], [3], [4]. Everywhere inside the fluid, its velocity $\mathbf{u}$ is determined from a velocity potential $\phi$, i.e.,

$$
\mathbf{u}=\nabla \phi
$$

where

$$
\nabla^{2} \phi=0
$$

everywhere in $D(t)$. The pressure condition on $\partial D(t)$ becomes

$$
\phi=\frac{A(t)}{2} z \bar{z} \quad \text { on } \partial D(t)
$$

where $A(t)=\frac{\rho \omega^{2}(t) k}{\mu}$, in which $\rho$ is the fluid density, $\mu$ its viscosity, and $k=b^{2} / 12$, where $b$ is the plate separation. A kinematic condition on each interface also requires that the local normal velocity of the boundary $V_{n}$ equal the local normal velocity of the fluid, i.e.,

$$
\text { u.n }=V_{n} .
$$

The evolution of the boundary is driven here by centrifugal effects.

Complex reformulation. Because the domain is simply connected, the general solution of (28) can be written

$$
\phi=\operatorname{Re}[g(z, t)],
$$

where $g(z, t)$ is analytic (in $z$ ) everywhere in $D(t)$. The time dependence of $g(z, t)$ derives from the fact that the boundary $D(t)$ is evolving in time. In terms of this analytic function, the kinematic boundary condition (30) can be written as

$$
\operatorname{Im}\left[\frac{d z}{d t} \bar{z}_{s}\right]=\operatorname{Im}\left[(u+i v) \bar{z}_{s}\right]=\operatorname{Im}\left[2 \phi_{\bar{z}} \bar{z}_{s}\right]=\operatorname{Im}\left[\bar{g}^{\prime}(\bar{z}) \bar{z}_{s}\right] \quad \text { on } \partial D(t) .
$$

Condition (29) can similarly be written, in complex notation, as the condition

$$
\phi=\frac{g(z)+\bar{g}(\bar{z})}{2}=\frac{A(t)}{2} z \bar{z} \quad \text { on } \partial D(t) .
$$

We now define the linear functional $\mathcal{L}$ :

$$
\mathcal{L}[h(z, t) ; D(t)] \equiv \iint_{D(t)} h(z, t) d x d y,
$$


where $h(z, t)$ is a function analytic everywhere in $D(t)$ and continuous on $\partial D(t)$.

THEOREM 4.1. In the free boundary problem just described, the following expression holds for the time evolution of $\mathcal{L}[h(z, t) ; D(t)]$ :

$$
\frac{d}{d t} \mathcal{L}[h(z, t) ; D(t)]=\mathcal{L}\left[h_{t}(z, t)+A(t) z h_{z}(z, t) ; D(t)\right],
$$

where $h(z, t)$ is analytic in $D(t)$ and continuous on $\partial D(t)$.

Proof. Using the complex form of Green's theorem in the plane,

$$
\mathcal{L}[h(z, t) ; D(t)]=\frac{1}{2 i} \oint_{D(t)} h(z, t) \bar{z} d z .
$$

Taking the time derivative,

$$
\frac{d \mathcal{L}[h(z, t) ; D(t)]}{d t}=\frac{1}{2 i} \oint_{\partial D(t)} h_{t} \bar{z} d z+h_{z} z_{t} \bar{z} d z+h\left(\bar{z}_{t} d z+\bar{z} d z_{t}\right) .
$$

However, from (32),

$$
\bar{z}_{t} d z=z_{t} d \bar{z}+2 \phi_{z} d z-2 \phi_{\bar{z}} d \bar{z}
$$

which, when substituted into (37), gives

$$
\begin{gathered}
\frac{d \mathcal{L}[h(z, t) ; D(t)]}{d t}=\frac{1}{2 i} \oint_{\partial D(t)} h_{t} \bar{z} d z+h_{z} z_{t} \bar{z} d z+h \bar{z} d z_{t}+h z_{t} d \bar{z} \\
+2 h\left(\phi_{z} d z-\phi_{\bar{z}} d \bar{z}\right) .
\end{gathered}
$$

However,

$$
h_{z} z_{t} \bar{z} d z+h \bar{z} d z_{t}+h z_{t} d \bar{z}=d\left(z_{t} h(z) \bar{z}\right)
$$

is a total (spatial) differential and therefore gives zero contribution, while it is known from (33) that on $\partial D(t)$

$$
\phi_{\bar{z}} d \bar{z}=d\left(\frac{A(t)}{2} z \bar{z}\right)-\phi_{z} d z
$$

which, when substituted into (40), gives

$$
\frac{d \mathcal{L}[h(z, t) ; D(t)]}{d t}=\frac{1}{2 i} \oint_{\partial D(t)} h_{t} \bar{z} d z+2 h\left(2 \phi_{z} d z-d\left(\frac{A(t)}{2} z \bar{z}\right)\right) .
$$

However, $2 \phi_{z}=g^{\prime}(z)$, which is analytic everywhere in $D(t)$, which leaves, after an integration by parts,

$$
\frac{d \mathcal{L}[h(z, t) ; D(t)]}{d t}=\frac{1}{2 i} \oint_{\partial D(t)} h_{t} \bar{z} d z+A(t) z h_{z} \bar{z} d z,
$$

which, using Green's theorem, provides the required result. 
4.2. A sintering viscous droplet. Now consider a very different physical problem: the unsteady evolution of a simply connected plane blob of very viscous (Stokes) fluid evolving purely under the effects of surface tension. Introducing a streamfunction $\psi(x, y)$ such that

$$
\mathbf{u}=\nabla^{\perp} \psi
$$

it is well known [5] that

$$
\nabla^{4} \psi=0 \quad \text { in } D(t)
$$

On the blob boundary the stress condition is

$$
-p n_{j}+2 e_{j k} n_{k}=\kappa n_{j},
$$

where $\kappa$ is the surface curvature. Again, the kinematic condition is that

$$
\mathbf{u . n}=V_{n}
$$

at each point on the interface. It is clear that this problem is mathematically (and physically) very different from the rotating Hele-Shaw problem.

Complex reformulation. The general solution of (45) at each instant can be written as

$$
\psi=\operatorname{Im}[\bar{z} f(z, t)+g(z, t)],
$$

where $f(z, t)$ and $g(z, t)$ are analytic everywhere in the fluid region $D(t)$. In terms of these Goursat functions, the following relations can easily be established:

$$
\begin{aligned}
p-i \omega & =4 f^{\prime}(z, t), \\
u+i v & =-f(z, t)+z \bar{f}^{\prime}(\bar{z}, t)+\bar{g}^{\prime}(\bar{z}, t), \\
e_{11}+i e_{12} & =z \bar{f}^{\prime \prime}(\bar{z}, t)+\bar{g}^{\prime \prime}(\bar{z}, t) .
\end{aligned}
$$

Defining $s$ to be the arclength traversed in an anticlockwise direction around the blob, it is known [5] that the stress boundary condition can be written in the form

$$
f(z, t)+z \bar{f}^{\prime}(\bar{z}, t)+\bar{g}^{\prime}(\bar{z}, t)=-i \frac{z_{s}}{2} .
$$

Using (52), the kinematic condition can be written as

$$
\operatorname{Im}\left[\left(z_{t}+2 f\right) \bar{z}_{s}\right]=-\frac{1}{2} .
$$

THEOREM 4.2. Under the evolution equations for Stokes flow, the following expression holds for the time evolution of the linear functional $\mathcal{L}$ defined over the simply connected time-evolving domain $D(t)$ :

$$
\frac{d}{d t} \mathcal{L}[h(z, t) ; D(t)]=\mathcal{L}\left[h_{t}(z, t)-2 f(z, t) h_{z}(z, t) ; D(t)\right],
$$

where $h(z, t)$ is analytic in $D(t)$ and continuous on $\partial D(t)$.

Proof. Using the complex form of Green's theorem in the plane, it is clear that

$$
\mathcal{L}[h(z, t) ; D(t)]=\frac{1}{2 i} \oint_{D(t)} h(z, t) \bar{z} d z .
$$


We now compute the time derivative of this quantity.

$$
\frac{d \mathcal{L}[h(z, t) ; D(t)]}{d t}=\frac{1}{2 i} \oint_{\partial D(t)} h_{t} \bar{z} d z+\frac{1}{2 i} \oint_{\partial D(t)} h_{z} z_{t} \bar{z} d z+\frac{1}{2 i} \oint_{\partial D(t)} h(\bar{z} d z)_{t} .
$$

It is necessary to compute $(\bar{z} d z)_{t}$ from the equations of motion. The stress condition provides that

$$
2 \bar{f}+2 \bar{z} \frac{d f}{d z}+2 \frac{d g}{d z}=i \bar{z}_{s}
$$

equivalently,

$$
2 \bar{f} d z+2 \bar{z} d f+2 d g=i \bar{z}_{s} d z=i d s
$$

using the fact that $z_{s} \bar{z}_{s}=1$. The kinematic condition can be written in the form

$$
\operatorname{Im}\left[\left(z_{t}+2 f\right) \bar{z}_{s}\right]=-\frac{1}{2}
$$

Or

$$
z_{t} \bar{z}_{s}+2 f \bar{z}_{s}-\bar{z}_{t} z_{s}-2 \bar{f} z_{s}=-i
$$

which is equivalent to

$$
z_{t} d \bar{z}+2 f d \bar{z}-\bar{z}_{t} d z-2 \bar{f} d z=-i d s .
$$

Combining (61) and (58) provides the expression

$$
(\bar{z} d z)_{t}=2 d g+2 d(\bar{z} f)+\bar{z} d z_{t}+z_{t} d \bar{z}
$$

which, when substituted into (56), gives the equation

$$
\begin{aligned}
\frac{d \mathcal{L}[h(z, t) ; D(t)]}{d t} & =\frac{1}{2 i} \oint_{\partial D(t)} h_{t} \bar{z} d z+2 h d g+2 h d(\bar{z} f) \\
& +\left[h_{z} z_{t} \bar{z} d z+h z_{t} \bar{z} d z+h z_{t} d \bar{z}\right] .
\end{aligned}
$$

Note, however, that the terms in square brackets represent a total (spatial) differential and therefore give a zero total contribution to the integral. Note also that the second term on the right-hand side of (63) gives zero contribution since both $h(z, t)$ and $g(z, t)$ are known to be analytic inside $D(t)$. Finally, using integration by parts, we obtain

$$
\frac{d \mathcal{L}[h(z, t) ; D(t)]}{d t}=\frac{1}{2 i} \oint_{\partial D(t)}\left(h_{t}-2 f h_{z}\right) \bar{z} d z
$$

which, with a final application of Green's theorem, completes the proof.

4.3. Evolution of the Cauchy transforms. It is clear that (35) and (54) have the same general form, i.e.,

$$
\frac{d}{d t} \mathcal{L}[h(z, t) ; D(t)]=\mathcal{L}\left[h_{t}(z, t)+\sigma(z, t) h_{z}(z, t) ; D(t)\right],
$$


where $\sigma(z, t)$ is analytic in $D(t)$. Now consider a point $\mathcal{Z}$ lying strictly outside the domain $D(t)$. Equations (35) and (54) can be used to derive the evolution of the Cauchy transforms $C(z, t)$ for each problem. Consider the special choice of (arbitrary) function $h(z, t)$ given by

$$
h_{C}(z, t ; \mathcal{Z})=\frac{1}{\pi} \frac{1}{z-\mathcal{Z}}
$$

where another complex variable $\mathcal{Z}$ enters as a parameter. This function is analytic (as a function of $z$ ), provided that $\mathcal{Z}$ is outside $D(t)$. Moreover, note that

$$
\mathcal{L}\left[h_{C}(z, t ; \mathcal{Z}) ; D(t)\right]=C(\mathcal{Z}, t) .
$$

Therefore to find the time evolution of $C(\mathcal{Z}, t)$ for each of the physical problems just discussed we can use Theorem 4.1 and Theorem 4.2, respectively:

$$
\begin{aligned}
\frac{\partial}{\partial t} \mathcal{L}\left[h_{C}(z, t ; \mathcal{Z}) ; D(t)\right]=\frac{\partial C(\mathcal{Z}, t)}{\partial t} & =-\frac{1}{\pi} \iint_{D(t)} \frac{\sigma(z)}{(z-\mathcal{Z})^{2}} d x d y \\
& \equiv-\frac{\partial}{\partial \mathcal{Z}} I(\mathcal{Z}, t)
\end{aligned}
$$

where

$$
\sigma(z, t)= \begin{cases}-2 f(z, t) & \text { for sintering Stokes flow, } \\ A(t) z & \text { for rotating Hele-Shaw flows. }\end{cases}
$$

Note crucially that $\sigma(z, t)$ is analytic inside $D(t)$ in both problems. Therefore both the rotating Hele-Shaw problem and the viscous sintering problem fall within the abstract class of geometry-driven free boundary problems considered in section 3 .

Remark. Corollary 3.2 immediately implies that both the rotating Hele-Shaw problem and the viscous sintering problem admit exact solutions for initial domains whose Cauchy transforms are meromorphic inside $D(0)$. These are the exact solutions already found by previous authors (cf. [14], [10]). The circulation theorem also holds for both problems; by taking $\gamma$ as successively encircling each pole inside $D(0)$, we find as many nontrivial conserved quantities for each problem as there are distinct poles (of any order). These conserved quantities are the same as those following from the "theorem of invariants" of [5]. Richardson [25] has also found these invariants using different methods involving conformal maps.

Remark. The circulation theorem (Theorem 3.3) leads to some previously unknown conserved quantities for the viscous sintering problem - in particular, even in situations in which the problem does not admit exact solutions, we have shown there still exists, in general, a set of nontrivial conserved quantities associated with the flow. Suppose an initial domain $D(0)$ has a Cauchy transform $C(z, 0)$ which has not only a finite distribution of poles inside $D(0)$ but also a finite set of branching singularities as shown in Figure 1. For this initial condition, there is no known exact solution for its evolution under the dynamics of viscous sintering; nevertheless, the dynamics preserves five nontrivial circulation-type quantities given in (26) with contours $\gamma_{j}$ shown in Figure 1.

5. Examples. We now examine various explicit examples in order to highlight the similarities and differences between these two problems. As a result, we gain some important new insights into both. 
5.1. Example 1. The following example highlights the similarities between the two physical problems stated above. Consider the evolution both in a rotating HeleShaw cell and due to viscous sintering of a blob described by a conformal mapping function having the rational function form

$$
z(\zeta, t)=\frac{R(t) \zeta}{\zeta^{2}-a^{2}(t)} .
$$

This corresponds to a Cauchy transform that is a rational function with two simple poles, at $z_{1}(t)$ and $z_{2}(t)=-z_{1}(t)$ say, inside $D(t)$.

For flow in a rotating Hele-Shaw cell, the evolution of the conformal map (70) of such blobs is given implicitly by the set of equations

$$
\begin{aligned}
\dot{z}_{1}(t) & =A(t) z_{1}(t), \\
z_{2}(t) & =-z_{1}(t), \\
\oint_{\gamma_{1}} C(z, t) d z & =\oint_{\gamma_{1}} C(z, 0) d z, \\
\oint_{\gamma_{2}} C(z, t) d z & =\oint_{\gamma_{2}} C(z, 0) d z,
\end{aligned}
$$

where $\gamma_{1}$ is chosen to be a small circular contour enclosing $z_{1}(t)$, while $\gamma_{2}$ similarly encloses $z_{2}(t)=-z_{1}(t)$. Note that, due to symmetry, $\oint_{\gamma_{1}} C(z, 0) d z=\oint_{\gamma_{2}} C(z, 0) d z$, so that (74) in fact constitutes just two independent equations for the two unknowns $a(t)$ and $R(t)$ in (70). The analogous equations for the viscous sintering problem are given by

$$
\begin{aligned}
\dot{z}_{1}(t) & =-2 f\left(z_{1}(t), t\right), \\
z_{2}(t) & =-z_{1}(t), \\
\oint_{\gamma_{1}} C(z, t) d z & =\oint_{\gamma_{1}} C(z, 0) d z, \\
\oint_{\gamma_{2}} C(z, t) d z & =\oint_{\gamma_{2}} C(z, 0) d z .
\end{aligned}
$$

Note how similar these equations are to those governing the rotating Hele-Shaw problem.

Using the above evolution equations for the rotating Hele-Shaw problem, it can be deduced that for a blob of area $\pi$ with the parameter $A(t)=1$ (so that the blob rotates with constant angular velocity) the evolution equations for $R(t)$ and $a(t)$ are given by

$$
\begin{aligned}
& a(t)=\sqrt{\frac{e^{-2 t}}{2 z_{0}^{2}}\left(1+\sqrt{1-4 z_{0}^{4} e^{4 t}}\right),} \\
& R(t)=\sqrt{\frac{\left(1-a(t)^{4}\right)^{2}}{\left(1+a(t)^{4}\right)}},
\end{aligned}
$$

where $z_{0}$ is the initial position of the point $z_{1}(t)=z_{0} e^{t}$. For a near-circular initial blob we take $z_{0}=\epsilon \ll 1$. A plot of the blob at a later time $t=5.3$ is shown in Figure 2. Note that after a finite time, the solution breaks down via the formation of two cusps in the free surface leading to a "splitting" event. The solution (70) for a sintering blob has been calculated by various previous authors and we refer the reader to those papers [14], [15], [25], [26] for plots of the solution in this case. 


\subsection{Example 2: The ellipse.}

5.2.1. Rotating Hele-Shaw cell. The example just given illustrates the mathematical similarities between the two problems. Next we consider the case of an ellipse, which illustrates some important differences between the two problems while at the same time showing the usefulness of the Cauchy transform approach.

Consider an initial elliptical blob of fluid (with major and minor axes coincident with the $x$ - and $y$ - axes) as it evolves both in a rotating Hele-Shaw cell and also in the context of viscous sintering. The Cauchy transform of an ellipse $(D(0)$, say) is given by

$$
C(z, 0)=R\left(z-\sqrt{z^{2}-z_{0}^{2}}\right)
$$

for some constants $R, z_{0}$. This Cauchy transform is not meromorphic inside the domain but has two square root branch-point singularities (and no other singularities in $D(0)$ ). Corollary 3.2 on the existence of exact solutions does not apply to this initial condition and, in general, we do not expect to find exact solutions to either problem for this initial condition. Nevertheless, we do have some information concerning the evolution of the elliptical blob. First, we know how the two square root branch points must evolve, i.e., we know the evolution of points $z_{1}(t)$ and $z_{2}(t)$ (say), with $z_{1}(0)=z_{0}$ and $z_{2}(0)=-z_{0}$. Second, the circulation theorem does pertain to this initial condition, and we immediately deduce the existence of a nontrivial conserved quantity given by

$$
\oint_{\gamma} C(z, t) d z
$$

where $\gamma$ is any contour surrounding the branch cut joining $z_{1}(t)$ and $z_{2}(t)$. In this case, this conserved quantity corresponds to the blob area.

It turns out that the rotating Hele-Shaw problem still admits an exact solution which is expressible explicitly in terms of a finite set of parameters. By virtue of the particular choice of $\sigma(z, t)$ in this case (7) can be integrated in closed form. Indeed, it can be checked that

$$
\begin{aligned}
C(z, t) & =e^{-A t} C\left(z e^{-A t}, 0\right) \\
& =R e^{-A t}\left(z e^{-A t}-\sqrt{z^{2} e^{-2 A t}-z_{0}^{2}}\right)
\end{aligned}
$$

is the unique solution of (7). This $C(z, t)$ is also the Cauchy transform of an ellipse but with different foci. For the viscous sintering problem, this will not be the case.

It is now expedient to consider the conformal map $z(\zeta, t)$ from the exterior of the unit circle to the exterior of the elliptical blob. Such a conformal map is given by

$$
z(\zeta, t)=\frac{a(t)}{\zeta}+b(t) \zeta
$$

where $a(t)$ and $b(t)$ are taken to be real for an ellipse that is symmetrical about both the $x$ - and $y$-axes. This is easily checked. It is possible to find a simple expression for the Cauchy transform $C(z, t)$ of the ellipse in terms of these conformal mapping parameters. To do this, note that on $|\zeta|=1$, 


$$
\begin{aligned}
\bar{z} & =a(t) \zeta+\frac{b(t)}{\zeta} \\
& =a\left(\frac{z}{b}-\frac{a}{b \zeta}\right)+\frac{b}{\zeta} \\
& =\frac{a}{b} z+\left(b-\frac{a^{2}}{b}\right) \frac{1}{\zeta} .
\end{aligned}
$$

Now observe that $\frac{b}{a} z$ is analytic inside the ellipse so that the Cauchy transform is given by

$$
C(z, t)=-\left(\frac{b^{2}-a^{2}}{b}\right) \frac{1}{\zeta}
$$

It can be shown that the area of the ellipse is given by $\pi\left(b^{2}-a^{2}\right)$, so choosing the area to be $\pi$, we deduce that $b^{2}-a^{2}=1$ for all times (this is a conserved quantity). Thus

$$
C(z, t)=-\frac{1}{b \zeta}
$$

However, from (84)

$$
\zeta=\frac{z+\sqrt{z^{2}-4 a b}}{2 b}
$$

so that

$$
C(z, t)=-\frac{1}{2 a b}\left(z-\sqrt{z^{2}-4 a b}\right) .
$$

For an ellipse that is symmetrical about the $x$ - and $y$-axes, there will be square root branch points at $z_{1}(t) \equiv 2 \sqrt{a b}, z_{2}(t)=-2 \sqrt{a b}$. However, the evolution of a branch point $z_{1}(t)$ is known to be $z_{1}(t)=z_{0} e^{t}$ (with $A=1$ ). Thus, the conformal mapping parameters satisfy the system

$$
\begin{aligned}
a(t)^{2} & =\frac{-1+\sqrt{1+\frac{z_{0}^{4} e^{4 t}}{4}}}{2}, \\
b(t) & =\sqrt{1+a(t)^{2}} .
\end{aligned}
$$

For a near-circular initial blob, we take $z_{1}(0)=z_{0}=\epsilon$, where $\epsilon \ll 1$. The subsequent shape of this initial near-circular blob at a later time $t=5.3$ is shown in Figure 3 . Note that this solution does not break down at finite time, but rather the solution exists globally in time, and the blob becomes increasingly elongated in the $x$-direction.

Remark. Entov, Etingof, and Kleinbock [10] considered the case of an elliptical bubble in a rotating Hele-Shaw cell (as opposed to an elliptical blob) and established that an initially elliptical bubble tends to a circle as $t \rightarrow \infty$. We have shown that an elliptical blob of fluid becomes infinitely elongated along one of its axes under evolution. The latter exact solution is new and is not reported in [10].

Remark. Comparison of Figures 2 and 3 illustrates that the rotating Hele-Shaw problem is ill-posed with respect to initial conditions. The initial value of $z_{0}$ in each problem can be made arbitrarily small so that the initial near-circular blobs in Figures 2 and 3 are virtually indistinguishable. The subsequent evolution of each 


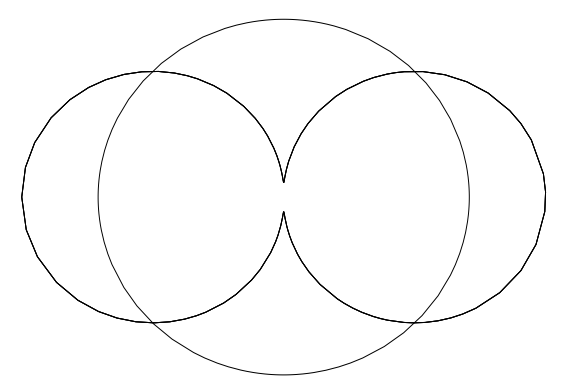

FIG. 2. Splitting of a near-circular blob in a rotating Hele-Shaw cell. Area $=\pi ; A(t)=1.0$; $z_{0}=0.01 ; t=0,5.3$.

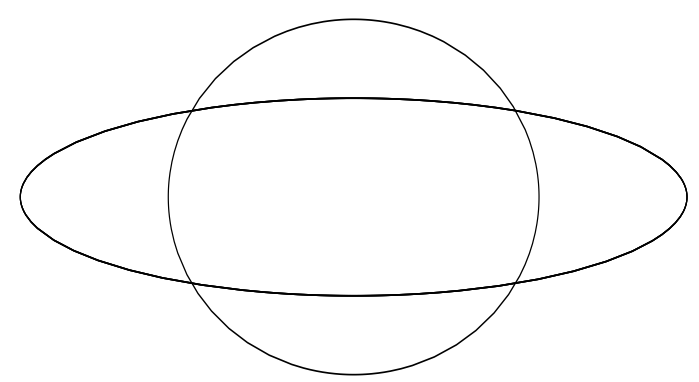

Fig. 3. Elongation of a near-circular blob in a rotating Hele-Shaw cell. Area $=\pi ; A(t)=1.0$; $z_{0}=0.01 ; t=0,5.3$.

blob is, however, drastically different: One breaks down (by "splitting") in finite time while the other exists globally in time and degenerates into a "line" as $t \rightarrow \infty$. Entov, Etingof, and Kleinbock [10] note the fact that blobs of fluid in a rotating Hele-Shaw cell can break down by "splitting" or by "cusp formation" at finite times (which they estimate for certain classes of initial condition). The above example shows that there are additional possibilities. There is therefore need for care in using this mathematical model to explain experiments in rotating Hele-Shaw cells. Some form of regularization of the model is needed to ascertain which, if any, of the solutions to this idealized mathematical problem are physically relevant. The ill-posedness of the standard (i.e., nonrotating) Hele-Shaw model is well known [17] and also possesses solutions which exist globally and remain smooth for all times (e.g., the SaffmanTaylor finger). However, the regularized problem has many subtleties and the smooth zero-regularization solutions need not necessarily be the physically relevant solutions in the limit that the regularization parameter is taken to zero [30]. We expect similar surprises to occur in the rotating Hele-Shaw problem.

5.2.2. A sintering elliptical blob. An initial elliptical blob does not remain elliptical under evolution due to viscous sintering; Hopper [16] demonstrated explicitly that the hypothesis that an initial ellipse circularizes through a sequence of ellipses is false. Using a boundary integral method, Kuiken [19] has numerically calculated the evolution due to viscous sintering of an initial elliptical blob given by

$$
x^{2}+10 y^{2}=1
$$


By $t \approx 2.0$ the ellipse has become very nearly circular, and examination of Kuiken's Figure 3 shows the evolution to be via a series of "ellipse-like" shapes (even though Hopper's result [16] shows that this is not strictly true). The calculation is computationally intensive, and there are various numerical difficulties involved which are discussed and analyzed in some detail by Kuiken [19].

In light of this research the following question seems natural: Exactly how accurate is an approximation of the dynamics of a sintering ellipse under the assumption that the evolution is via a series of ellipses? This question does not appear to have been previously examined in the literature, but our present methods suggest a way to do this.

Given that the fluid volume is conserved, the only unknown parameter in such an approximating solution would be the positions of the branch points of the Cauchy transform. However, Theorem 3.1 provides exactly the ordinary differential equation needed to determine the evolution of these singularities, specifically,

$$
\sigma\left(z_{1}(t), t\right)=-2 f\left(z_{1}(t), t\right) .
$$

Denoting the conformal map from the unit circle to an ellipse by the map $z(\zeta, t)$, it can be shown [5] that

$$
-2 f(z, t)=z_{t}(\zeta, t)-\zeta I(\zeta, t) z_{\zeta}(\zeta, t)
$$

where the Poisson integral $I(\zeta, t)$ is given by

$$
I(\zeta, t)=\frac{1}{4 \pi i} \oint_{\left|\zeta^{\prime}\right|=1} \frac{d \zeta^{\prime}}{\zeta^{\prime}}\left(\frac{\zeta^{\prime}+\zeta}{\zeta^{\prime}-\zeta}\right) \frac{1}{z_{\zeta}^{1 / 2}(\zeta, t) \bar{z}_{\zeta}^{1 / 2}\left(\zeta^{-1}, t\right)} .
$$

Using the fact that $z_{1}(t)=z\left(\zeta_{1}(t), t\right)$, where $\zeta_{1}(t)$ is the preimage of the branch point $z_{1}(t)$, we deduce

$$
\dot{z}_{1}(t)=z_{t}\left(\zeta_{1}(t), t\right)+\dot{\zeta}_{1}(t) z_{\zeta}\left(\zeta_{1}(t), t\right)
$$

but from (94) and (13)

$$
\dot{z}_{1}(t)=-2 f\left(z_{1}(t), t\right)=z_{t}\left(\zeta_{1}(t), t\right)-\zeta_{1}(t) I\left(\zeta_{1}(t), t\right) z_{\zeta}\left(\zeta_{1}(t), t\right) ;
$$

thus the evolution of $\zeta_{1}(t)$ is deduced to be

$$
\dot{\zeta}_{1}(t)=-\zeta_{1}(t) I\left(\zeta_{1}(t), t\right)
$$

From [20], the mapping from the unit circle to an ellipse with semimajor and semiminor axes $a$ and $b$, respectively, is given by

$$
\zeta=\sqrt{\kappa} \operatorname{sn}\left(\frac{2 K}{\pi} \sin ^{-1}\left(\frac{z}{\sqrt{a^{2}-b^{2}}}\right)\right),
$$

where

$$
\begin{aligned}
e^{i \pi \tau} & =\left(\frac{a-b}{a+b}\right)^{2}, \\
\kappa & =\left(\frac{\Theta_{2}(\tau)}{\Theta_{3}(\tau)}\right)^{2}, \\
K & =F\left(\kappa, \frac{\pi}{2}\right),
\end{aligned}
$$




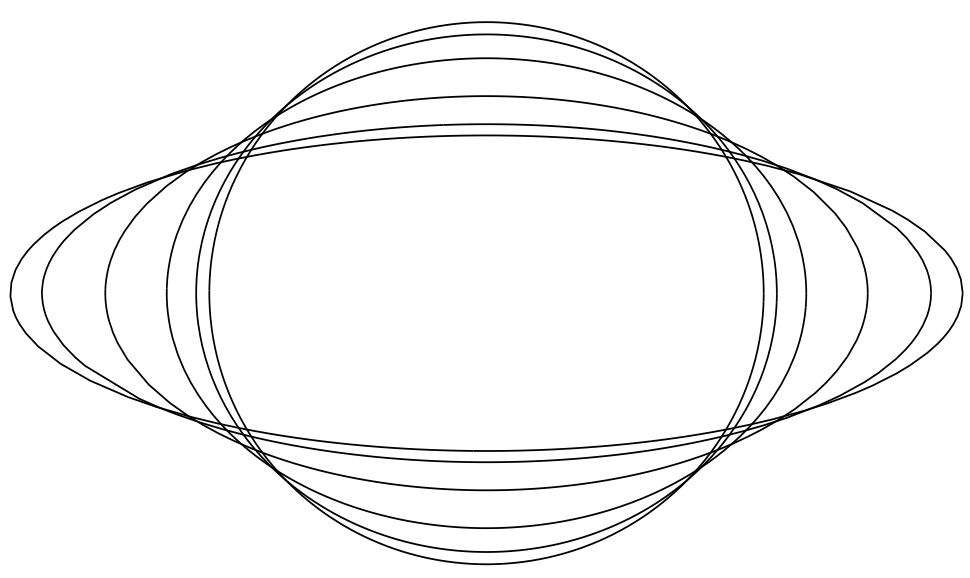

FIG. 4. Sintering ellipse: $t=0.0,0.2,0.5,1.0,1.5,2.0$ (cf. Figure 3 of [19]).

where $F$ denotes an elliptical integral of the first kind. The two branch points of the Cauchy transform are at points $\pm \sqrt{a^{2}-b^{2}}$.

The following numerical procedure was adopted to compute required approximation: a simple explicit first-order Euler time-stepping integration scheme was used to solve the differential equation (98) for $\zeta_{1}(t)$. This equation, along with the conservation of area result, stated as $\pi a b=\mathcal{A}$ (where $\mathcal{A}$ is the initial area), is enough to determine the evolution of parameters $a(t)$ and $b(t)$ describing the ellipse at each time. Initial parameters corresponding to the same initial ellipse (92) studied by Kuiken [19] were used. The subsequent (approximate) shapes at times $t=0.2,0.5,1.0,1.5,2.0$ are plotted in Figure 4. These are exactly the subsequent times plotted by Kuiken [19]. Kuiken did not include in his paper any numerical data, and thus we have not been able to compare the results quantitatively to any degree. However, a simple visual comparison by superposition of (suitably rescaled) versions of Figure 4 with Figure 3 of [19] shows remarkable closeness of the successive curves throughout the evolution. This closeness of the curves appears to improve as the solution evolves. Most importantly, both computations retrieve the same nondimensionalized time (i.e., $t \simeq 2.0$ ) by which the system has very nearly equilibrated to the asymptotic circular domain. This is perhaps the most important quantity from a physical point of view, and it is well captured by our two-dimensional approximation to this complicated free boundary problem. Even more, our approximation method encounters none of the numerical problems of the full boundary integral method [19].

6. Summary. The analogy between Hele-Shaw free boundary problems and the inverse gravitational problem has been a useful one and has led to many physical and mathematical advances in the study of free boundary problems. This includes results on the existence, uniqueness, and regularity of solutions (e.g., [18], [28], [29], and references therein). A generalized inverse gravitational problem of a specific type has been introduced in this paper and has been shown to be analogous to both the rotating Hele-Shaw problem and the viscous sintering problem. These mathematical connections have led to new results and insights presented both here and elsewhere [8]. Also of interest is the question of whether there exist other free boundary problems of physical importance which fall into the class of problems considered here (i.e., for other choices of $\sigma(z, t))$. It is important to point out that the evolution of the Cauchy 
transform in the case when the rotating Hele-Shaw problem has nonzero surface tension is not given by a conservation-type equation of the simple form considered in this paper, and therefore none of the conclusions reached in this paper pertain to this case.

Extension of the approach to cases in which the fluid domains are simply connected but unbounded (infinite) can be made and similar results obtained (e.g., regarding exact solutions). Finally, the Cauchy transform is readily defined for multiply connected domains of arbitrary finite connectivity. The formulation of the two physical problems considered herein in terms of Cauchy transforms is therefore expected to provide important insights into finding exact solutions to these two problems for fluid regions with more complicated topological structure than the simply connected domains treated here.

\section{REFERENCES}

[1] M. Ablovitz and A.S. Fokas, Complex Variables, Cambridge University Press, London, 1997.

[2] L. Carrillo, F.X. Magdeleno, J. Casademunt, and J. Ortin, Experiments in a rotating Hele-Shaw cell, Phys. Rev. E, 54 (1996), pp. 6260-6267.

[3] L. Carrillo, J. Soriano, and J. Ortin, Radial displacement of a fluid annulus in a rotating Hele-Shaw cell, Phys. Fluids, 11 (1999), pp. 778-785.

[4] L. Carrillo, J. Soriano, AND J. Ortin, Interfacial instabilities of a fluid annulus in a rotating Hele-Shaw cell, Phys. Fluids, 12 (2000), pp. 1685-1698.

[5] D.G. Crowdy and S. TAnveer, A theory of exact solutions for plane viscous blobs, J. Nonlinear Sci., 8 (1998), pp. 261-279.

[6] D.G. Crowdy and S. TAnveer, A theory of exact solutions for annular viscous blobs, J. Nonlinear Sci., 8 (1998), pp. 375-400.

[7] D.G. Crowdy, A note on viscous sintering and quadrature identities, European J. Appl. Math., 10 (1999), pp. 623-634.

[8] D.G. Crowdy, A theory of exact solutions for the evolution of a fluid annulus in a rotating Hele-Shaw cell, Quart. Appl. Math., to appear.

[9] P.J. DAvis, The Schwarz Function and Its Applications, Carus Math. Monogr. 17, Mathematical Association of America, Washington, DC, 1974.

[10] V.M. Entov, P.I. Etingof, And D.YA. Kleinbock, On nonlinear interface dynamics in HeleShaw flows, European J. Appl. Math., 6 (1995), pp. 399-420.

[11] V. Entov and P.I. Etingof, Bubble contraction in Hele-Shaw cells, Quart. J. Mech. Appl. Math., 44 (1991), pp. 507-535.

[12] V.M. Entov And P.I. Etingof, Viscous flows with time-dependent free boundaries in a nonplanar Hele-Shaw cell, European J. Appl. Math., 8 (1997), pp. 23-35.

[13] P.I. Etingof, Inverse problems of potential theory and flows in porous media with timedependent free boundaries, Comput. Math. Appl., 22 (1991), pp. 93-99.

[14] R.W. Hopper, Coalescence of two equal cylinders: Exact results for creeping viscous flow driven by capillarity, J. Am. Ceram. Soc., 67 (1985), pp. C262-264.

[15] R.W. Hopper, Plane Stokes flow driven by capillarity on a free surface, J. Fluid Mech., 213 (1990), pp. 349-375.

[16] R.W. Hopper, Plane Stokes flow driven by capillarity on a free surface. Part 2: Further developments, J. Fluid Mech., 230 (1991), pp. 355-364.

[17] S.D. Howison, Fingering in Hele-Shaw cells, J. Fluid Mech., 167 (1986), pp. 439-453.

[18] L. Karp AND A.S. Margulis, Newtonian potential theory for unbounded sources and applications to free boundary problems, J. Anal. Math., 70 (1996), pp. 1-63.

[19] H.K. Kuiken, Viscous sintering: The surface-tension-driven flow of a liquid form under the influence of curvature gradients at its surface, J. Fluid Mech., 214 (1990), pp. 503-515.

[20] H. Kober, A Dictionary of Conformal Representations, Dover, New York, 1957.

[21] G. Prokert, On the existence of solutions in plane quasistationary Stokes flow driven by surface tension, European J. Appl. Math., 6 (1995), pp. 539-558.

[22] S. Richardson, Hele-Shaw flows with a free boundary produced by injection of fluid in a narrow channel, J. Fluid Mech., 56 (1972), pp. 609-618.

[23] S. Richardson, Some Hele-Shaw flows with time-dependent free boundaries, J. Fluid Mech., 102 (1981), pp. 263-278. 
[24] S. Richardson, Hele-Shaw flows with time-dependent free boundaries involving injection through slits, Stud. Appl. Math, 87 (1992), pp. 175-194.

[25] S. Richardson, Two dimensional slow viscous flows with time dependent free boundaries driven by surface tension, European J. Appl. Math., 3 (1992), pp. 193-207.

[26] S. Richardson, Two dimensional Stokes flows with time dependent free boundaries driven by surface tension, European J. Appl. Math., 8 (1997), pp. 311-329.

[27] L.W. Schwartz, Instability and fingering in a rotating Hele-Shaw cell or porous medium, Phys. Fluids, 1 (1989), pp. 167-169.

[28] F.R. Tian, A Cauchy integral approach to Hele-Shaw problems with a free boundary: The zero surface tension case, Arch. Ration. Mech. Anal., 135 (1996), pp. 175-196.

[29] F.R. TIAn, On the breakdown of Hele-Shaw solutions with non-zero surface tension, J. Nonlinear Sci., 5 (1995), pp. 479-484.

[30] S. Tanveer, Surprises in viscous fingering, J. Fluid Mech., 409 (2000), pp. 273-308. 